\title{
mRNA Decapping Factors LSM1 and PAT Paralogs Are Involved in Turnip Mosaic Virus Viral Infection
}

\author{
Zhangli Zuo, Milena Roux, Eleazar Rodriguez, and Morten Petersen ${ }^{\dagger}$ \\ Department of Biology, Faculty of Science, University of Copenhagen, Copenhagen, Denmark
}

Accepted 27 October 2021.

\begin{abstract}
Turnip mosaic virus is a devastating potyvirus infecting many economically important brassica crops. In response to this, the plant host engages its RNA silencing machinery, involving AGO proteins, as a prominent strategy to restrain turnip mosaic virus (TuMV) infection. It has also been shown that the mRNA decay components DCP2 and VCS partake in viral infection suppression. Here, we report that the mRNA decapping components LSM1, PAT1, PATH1, and PATH2 are essential for TuMV infection. More specifically, lsmla/ lsm $1 b$ double mutants and pat1/path1/path2 triple mutants in summ2 background exhibit resistance to TuMV. Concurrently, we observed that TuMV interferes with the decapping function of LSM1 and PAT proteins as the mRNA-decay target genes $U G T 87 A 2$ and $A S L 9$ accumulate during TuMV infection. Moreover, as TuMV coat protein can be specifically found in complexes with PAT proteins but not LSM1, this suggests that TuMV "hijacks" decapping components via PAT proteins to support viral infection.
\end{abstract}

Keywords: LSM1, mRNA decapping, PAT paralogs, Turnip mosaic virus

Turnip mosaic virus belongs to the Potyvirus genus, which accounts for over $12.5 \%$ of all viruses known to infect plant species and causes significant losses in many crops (Fauquet and Fargette 2005; Wylie et al. 2017). The turnip mosaic virus (TuMV) genome is a single positive RNA strand of 9,830 nt covalently linked to VPg (viral genome-linked protein) in its $5^{\prime}$ end and contains a 3 '-poly(A) tail. The genome encodes two polyproteins processed into 11 mature proteins: P1, HC-Pro, P3, P3N-PIPO, 6K1, CI, 6K2, VPg, NIa, NIb, and CP (coat protein) (Olspert et al. 2015; Rodamilans et al. 2015; Urcuqui-Inchima et al. 2001; White, 2015).

\section{${ }^{\dagger}$ Corresponding author: M Petersen; shutko@bio.ku.dk}

Current address for Milena Roux: Novo Nordisk, Regulatory Affairs Durable Devices and Needles, Søborg, Denmark.

Funding: This work was supported by the Independent Research Fund Denmark (9041-00014B) to M. Petersen and a Ph.D. scholarship from China Scholarship Council (201504910714) to Z. Zuo.

*The $\boldsymbol{e}$-Xtra logo stands for "electronic extra" and indicates there are supplementary materials published online.

The author(s) declare no conflict of interest. distributed under the CC BY-NC-ND 4.0 International license.
Plant RNA silencing is the main antiviral process triggered by endogenous or exogenous double-stranded (ds)RNA, which is processed by DCLs (Dicer-like) into 21- to 24-nt small interfering (si)RNAs (Ding and Voinnet 2007; Pumplin and Voinnet 2013). These siRNAs subsequently associate with AGO proteins to form the RISC (RNA-induced silencing complex), which mediates degradation of viral RNA (Garcia-Ruiz et al. 2010, 2015; Qu et al. 2008). Consequently, large amounts of siRNAs derived from viral RNA can be detected in infected plants (Ding and Voinnet 2007). In response, TuMV has evolved silencing suppressors such as HCPro and VPg to interfere with host antiviral silencing. HCPro suppresses silencing by binding siRNAs and inhibiting DCL2 and DCL4 functions (Lin et al. 2007; Kung et al. 2014; Poque et al. 2018). Deletion of HCPro renders TuMV noninfectious but its infectivity is restored in RNA silencing-deficient mutants such as $d c l 2$ and $d c l 4$ (Garcia-Ruiz et al. 2010). On the other hand, VPg promotes autophagic and proteasomal degradation of SGS3 (Suppressor of Gene Silencing 3), a key component of the RNA silencing response (Cheng and Wang 2017).

In addition to RNA silencing, mRNA decay also regulates mRNA turnover. mRNA decay and RNA silencing are thought to be antagonistic processes that may compete for RNA substrates ( $\mathrm{Li}$ and Wang 2018). mRNA decay is initiated by deadenylation, removal of the $3^{\prime}$-poly(A) tail, and degradation either via $3^{\prime}-5^{\prime}$ exosomal exonucleases or via the $5^{\prime}-3^{\prime}$ exoribonuclease activity of the decapping complex (Garneau et al. 2007). mRNA decapping and 5'-3' decay occur in distinct cytoplasmic foci, termed processing bodies (PB). The decapping complex includes the decapping enzymes DCP1 (Decapping 1) and DCP2, which form a heterodimer and remove the cap along with other decapping components, including DCP5, DHH1 (DExD/ H-box RNA helicase), VCS (Varicose), LSM1-7 (Sm-Like 1-7) heptameric complex, PAT1 (Protein Associated with Topoisomerase II 1) and exoribonuclease XRN4 (Garneau et al. 2007; Xu and Chua 2011). The well-conserved protein PAT1 promotes PB assembly and activates decapping by binding mRNA and recruiting other factors including DHH1, the LSM1-7 complex, DCP5, VCS, DCP1/2, and XRN1 (Charenton et al. 2017; Chowdhury et al. 2014; Ozgur et al. 2010; Pilkington and Parker 2008). It has been shown that $d c p 2$ mutants exhibit an enhanced RNAsilencing phenotype and this can be restored by TuMV infection; this indicates that mRNA decay affects TuMV infection (Thran et al. 2012). A recent study found that VPg interferes with the interaction between DCP1 and DCP2 by switching DCP2 localization from cytoplasmic to nuclear and that HCPro interacts with XRN4 and suppresses its activity (Li and Wang 2018). While dcp2 mutants exhibit susceptibility to TuMV (Li and Wang 2018), xrn4 mutants exhibit either resistance or susceptibility to TuMV in different conditions ( $\mathrm{Li}$ and Wang 2018; Vogel et al. 2011). VCS was found to be an essential component of potyvirus potato 
virus A (PVA)-induced RNA granules, such that silencing VCS in host plants leads to resistance to PVA (Hafrén et al. 2015). All these findings indicate that mRNA decay components play different roles during TuMV infection. Nothing is known about the role of other mRNA decapping components, such as PAT proteins and LSM1 during TuMV infection. Intriguingly, yeast PAT1, LSM1-7, and DHH1 are required for brome mosaic virus (BMV) protein translation and RNA replication (Jungfleisch et al. 2015) and Ty1 retrotransposition during assembly of retrotransposition-competent virus-like particles (Checkley et al. 2010). Depletion of human PATL1 and LSM1 directly and specifically inhibits hepatitis $\mathrm{C}$ virus (HCV) RNA translation and replication (Scheller et al. 2009). This suggests that mRNA decapping components PAT and LSM1 may have a similar function during potyviral infection in Arabidopsis.

In Arabidopsis, LSMI is duplicated and codes for the pair of functionally redundant proteins LSM1A and LSM1B. Arabidopsis also encodes three PAT paralogs, PAT1, PATH1, and PATH2. PAT1 is under surveillance by the resistance protein SUMM2 (Suppressor of MKK1 MKK2 2), and loss of PAT1 function activates SUMM2 and triggers autoimmunity (Roux et al. 2015; Zhang et al. 2012). Thus, PAT paralog functions are best studied in a summ 2 mutant background (Roux et al. 2015; Zhang et al. 2012). In this context, we recently reported that all three PAT paralogs target ASL9 to enable apical hook and lateral root formation (Zuo et al. 2019) and that PAT1 also functions in response to the phytohormone abscisic acid (ABA) and osmotic stress, being involved in the decapping of the canonical ABA responsive gene COR15A (Zuo et al. 2021). Here, we report that all three PAT paralogs and LSM1 are needed for TuMV infection. TuMV triggers accumulation of PAT1, LSM1 and their decapping target mRNAs, which indicates that TuMV alters or inhibits the function of mRNA decapping components. We also detected PAT proteins, but not LSM1, in complex with TuMV CP protein. These data indicates that PAT proteins are hijacked by CP to interfere with PAT and LSM1 processes to support viral replication.

While $d c p 2$ mutants exhibit increased susceptibility and $v c s$ mutants increased resistance upon TuMV infection, $x r n 4$ susceptibility seems conditional (Hafrén et al. 2015; Li and Wang 2018; Vogel et al. 2011). To evaluate if other decapping components are also involved in potyvirus infection, we infected lsmla/lsm1b, dhh1, and pat1/path1/path2/summ2 mutants with the infectious clone pCB-TuMV-GFP (Garcia-Ruiz et al. 2010). Since PAT1 is under SUMM2 surveillance, we generated pat single, double, and triple mutants in summ 2 backgrounds for further studies (Roux et al. 2015; Zuo et al. 2019). We used eif4e as a negative control since eIF4E is required for potyvirus translation initiation (Duprat et al. 2002). Detection of TuMV $\mathrm{CP}$ using anti-TuMV $\mathrm{CP}$ in protein isolated from systemic infected leaves revealed less TuMV CP in lsmla/lsmlb compared with Arabidopsis thaliana ecotype Columbia (Col-0) and dhhl 14 days postinfection (dpi) (Fig. 1A). Mutants with all three PAT genes knocked out also had much less TuMV CP compared with single and double pat mutants, which had TuMV $\mathrm{CP}$ levels similar to Col-0 and summ2 (Fig. 1B). To better visualize the viral infection, we also examined and quantified the green fluorescent protein (GFP) fluorescence in $l s m l a / l s m l b$ and the complementation line $c$-lsmla (Perea-Resa et al. 2016) (Supplementary Fig. S1A and B) and multiple pat mutants (Supplementary Fig. S1C and D). While GFP could be detected in local infected leaves, we barely observed any GFP signal in lsmlal $l s m l b$ systemic leaves. However, massive GFP signal was detected in $c$-lsmla systemic leaves, similar to levels observed in Col-0 leaves (Supplementary Fig. S1A and B). Unlike TuMV $\mathrm{CP}$ immunoblotting analysis in which pat single and double mutants showed no difference from summ2, pat1 single and pat double mutants exhibited less GFP signal in systemic leaves than summ2, while the pat triple mutant only exhibited GFP signal in local leaves (Supplementary Fig. S1C and D). This indicates that the three PAT proteins are functionally redundant and additive during TuMV infection. Together, these findings suggest that decapping components LSM1 and PAT paralogs function differently from DCP2 and DHH1 but similarly to VCS, which is needed for viral infection.

To evaluate if $L S M 1$ and PAT genes are quantitative determinants of TuMV infection severity over time, we infected lsmla/ $l s m 1 b$, pat single, double, and triple mutants with TuMV and checked viral accumulation at $28 \mathrm{dpi}$. We observed lighter disease symptoms, including less stunting and decelerated leaf senescence, in pat single and double mutants than in Col-0 and summ2, while pat triple mutants appeared almost unaffected (Fig. 2A and B; Supplementary Fig. S2A and B). Double mutant lsmla/ $l s m l b$ also showed mild symptoms, while $c$-lsmla exhibited disease symptoms similar to Col-0 (Fig. 2A and C; Supplementary Fig. S2C). TuMV CP accumulation revealed that pat single, double, and triple mutants all had increased resistance to TuMV compared with Col-0 and summ2 (Supplementary Fig. S2A). These data confirms that the PAT proteins function in TuMV propagation in an additive way and the quantity of PAT proteins affects viral accumulation (Fig. 2; Supplementary Fig. S2).

Since LSM1 and PAT are needed for TuMV infection, we examined whether their protein levels were affected by TuMV.

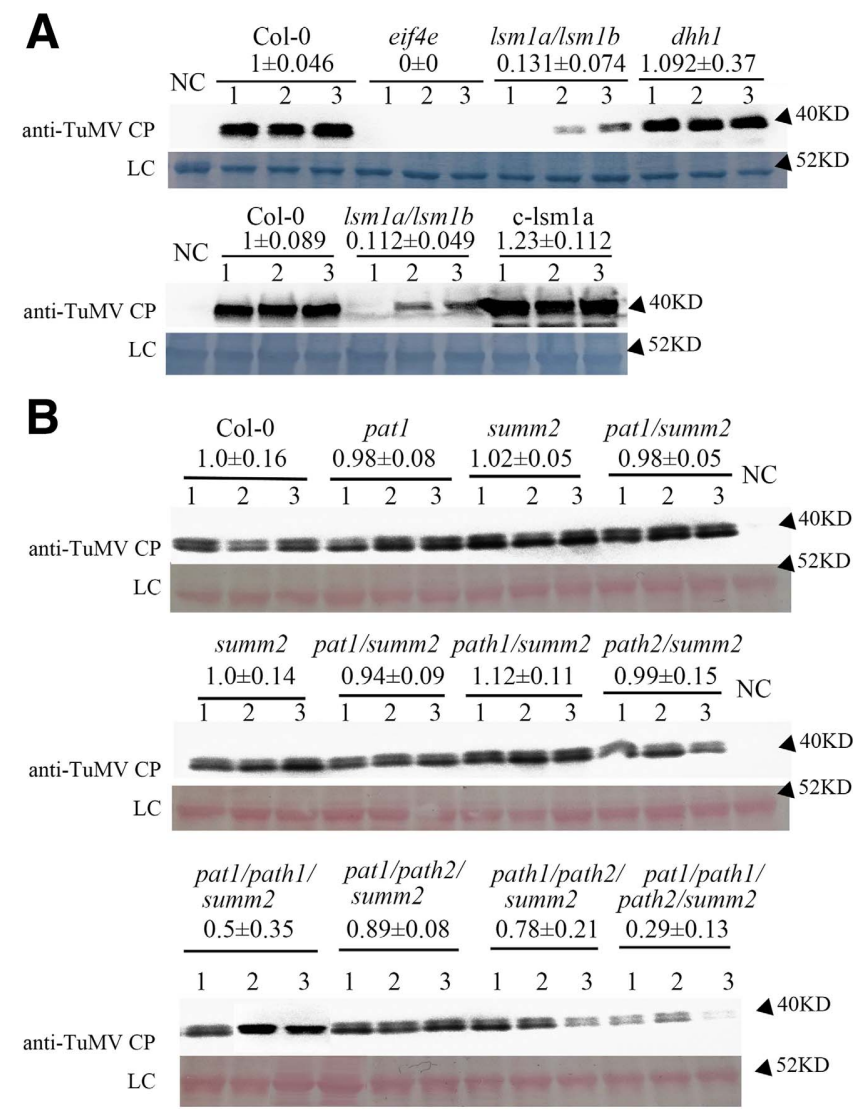

Fig. 1. mRNA decay mutants $l$ sm 1 a/lsm 1 b and pat1/path1/path2/summ 2 exhibit resistance to turnip mosaic virus (TuMV). A, Quantification of viral coat protein $(\mathrm{CP})$ levels in Col-0, eif4e, lsmla/lsm1b, dhhl, and c-lsm1a and B, summ2, pat1/summ2, path1/summ2, path2/summ2, pat1/ path1/summ2, pat1/path2/summ2, path1/path2/summ2, and pat1/path1/ path $2 /$ summ 2 at 14 days postinfection (dpi). All values represent means \pm standard error, $n=9$. The experiments were repeated three times. The amido black staining (A) and Ponceau S staining(B) of Rubisco serve as a loading control (LC). 
We infected transgenic plants expressing LSM1-GFP and Venus-PAT1 driven by their own promoters in $l s m l a / l s m l b$ or pat1 mutant backgrounds. We detected more LSM1 and PAT1 accumulation in TuMV-infected plants (Fig. 3A). Given the function of PAT and LSM1 proteins in mRNA decay, it is possible that viral RNA degradation is affected in $l s m l a / l s m l b$ and pat1/path1/path2/summ 2 mutants. To test this, we blocked transcription using cordycepin and examined the degradation rate of viral RNA in these lines, but only small differences were observed in respect to Col-0 (Supplementary Fig. S3). To study the possibility that the degradation of the mRNA decay targets is disrupted upon TuMV infection, we checked the expression

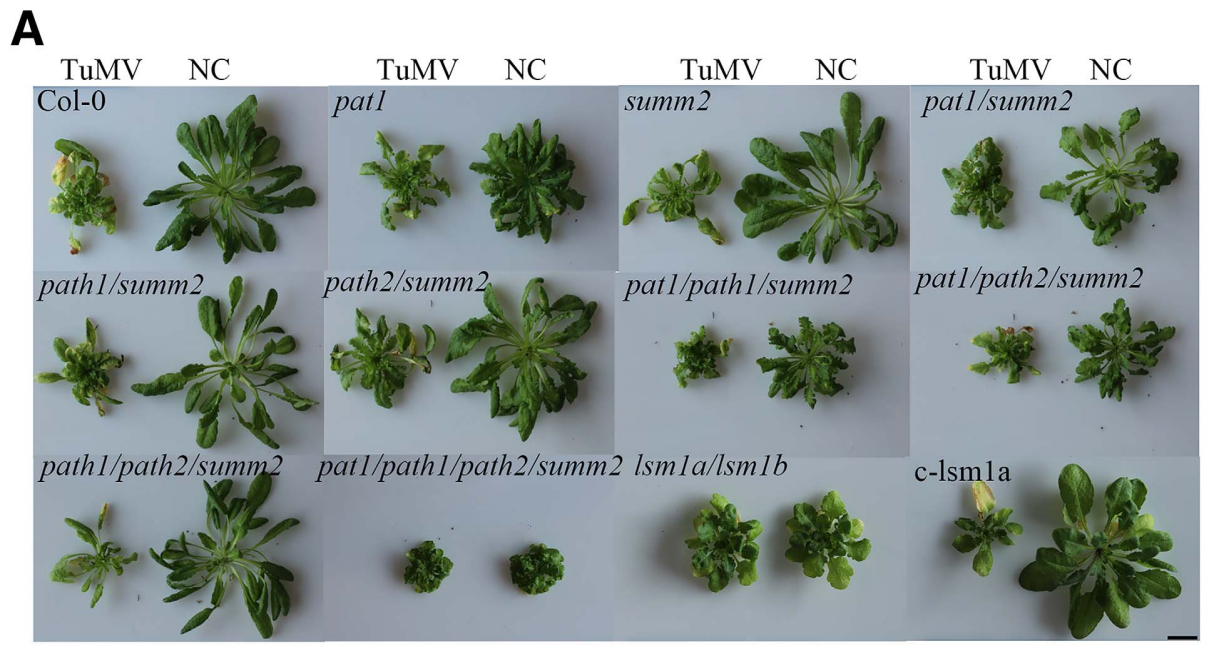

B

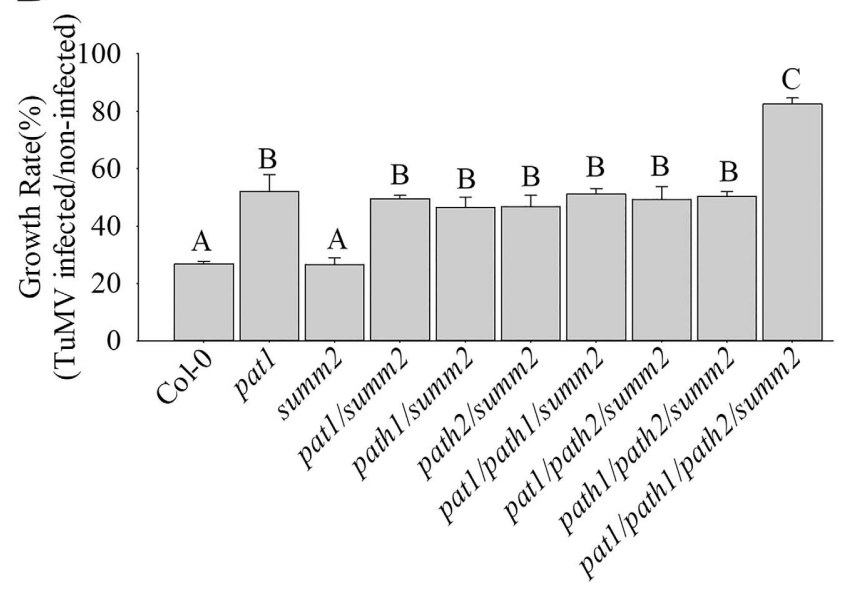

C

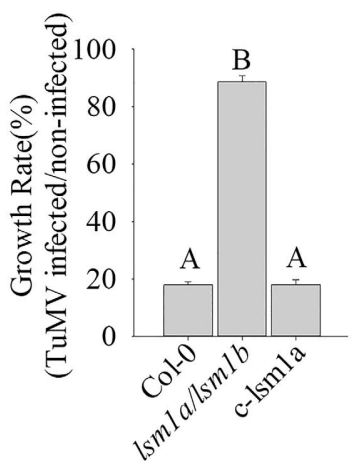

Fig. 2. The pat single, double, and triple mutants and $l s m l a / l s m l b$ are resistant to turnip mosaic virus (TuMV) in longer terms. A, Virus-induced symptoms and $\mathbf{B}$, plant growth rate in pat single double and triple mutants and $\mathbf{C}, l s m l a / l s m l b, c$-lsmla and control plants at 28 days postinfection $(\mathrm{dpi})$. Scale bar in $\mathrm{A}=2$ $\mathrm{cm}$. All values represent means \pm standard deviation, $n=3$. Bars marked with the same letter are not significantly different from each other $(P$ value $>0.05)$.

A

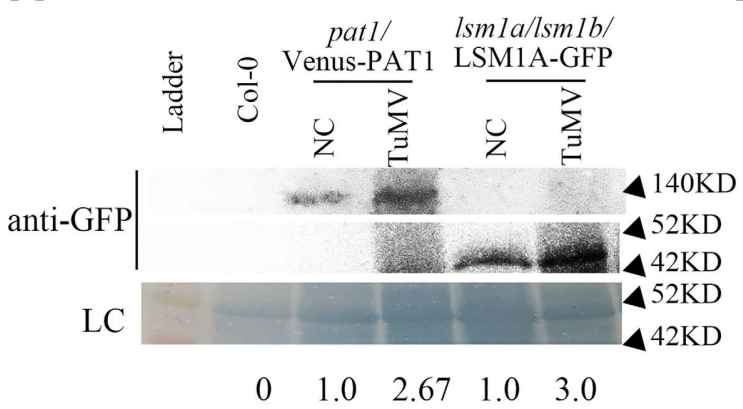

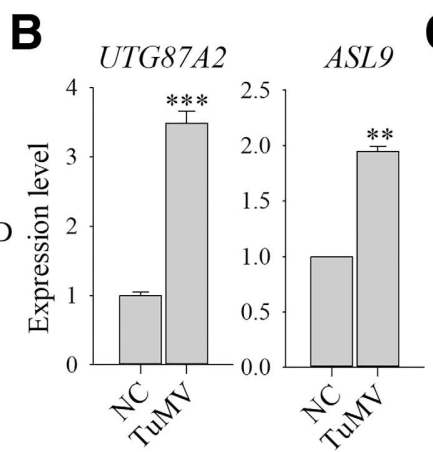

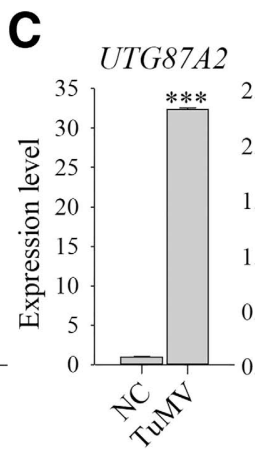

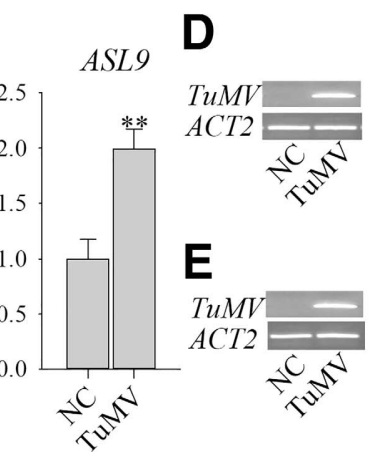

Fig. 3. Turnip mosaic virus (TuMV) interferes with the mRNA decay function of LSM1 and PAT1. A, Immunoblot analysis of LSM1 and PAT1 accumulation in $c$-lsmla and pat1/Venus-PAT1 plants at 14 days postinfection (dpi) with TuMV. Anti-GFP (green fluorescent protein) bands indicate accumulation of Venus-PAT1 (upper panel) and LSM1-GFP (lower panel), the amido black staining of Rubisco serves as a loading control (LC). Venus-PAT1 and LSM1-GFP signals in negative control (NC) plants are normalized to 1, respectively. B, UGT87A2 and ASL9 accumulated in Col-0 at 14 dpi and C, 28 dpi with TuMV. Quantitative PCR was used to check transcript levels; error bars indicate standard error $(n=3)$. Bars marked with two asterisks (**) are statistically significant $(P$ value $<0.01)$ and bars marked with three asterisks $(* * *)$ are statistically extremely significant $(P$ value $<0.001)$. D, Viral RNA accumulation serves as infection marker 14 dpi and E, 28 dpi separately. 
level of mRNA decapping target genes UGT87A2 and ASL9 at 14 and 28 dpi in TuMV-infected plants. Surprisingly, both UGT87A2 and ASL9 transcripts accumulated in TuMV infected plants at these two timepoints (Fig. 3B, C, D, and E), indicating that the function of PAT and LSM1 in mRNA decay were altered or inhibited by TuMV infection.

PAT1 and LSM1-7 are required for BMV and HCV RNA replication and translation in yeast and human cells and Ty1 retrotransposition during assembly of retrotransposition-competent virus-like particles (Checkley et al. 2010; Jungfleisch et al. 2015; Scheller et al. 2009). To investigate if LSM1 and PAT paralogs are involved in processes related to $\mathrm{CP}$, such as viral assembly, we transiently expressed hemagglutinin (HA)-tagged PAT proteins PAT1-HA, PATH1-HA, PATH2-HA, and LSM1-HA with a TuMV infectious clone and performed coimmunoprecipitation assays. TuMV CP could be detected in all three PAT-HA immunoprecipitates but not the LSM1-HA or SUMM2-HA (negative control) immunoprecipitates (Fig. 4), indicating that PATs are directly targeted by TuMV CP (e.g., for processes such as viral encapsidation) while LSM1 is targeted indirectly, possibly through PAT paralogs.

LSM1-7 and PAT1 form complexes and promote mRNA decapping together with other decapping components (Tharun and Parker 2001; Tharun et al. 2000). As Arabidopsis PAT paralogs PAT1, PATH1, and PATH2 have all been shown to form complexes with LSM1, it is not surprising that $l s m 1 a / l s m 1 b$ and pat triple mutants exhibit similar resistance phenotypes to TuMV infection (Figs. 1 and 2; Supplementary Figs. S1 and S2). Since TuMV interferes with mRNA decay (Fig. 3) and we detected

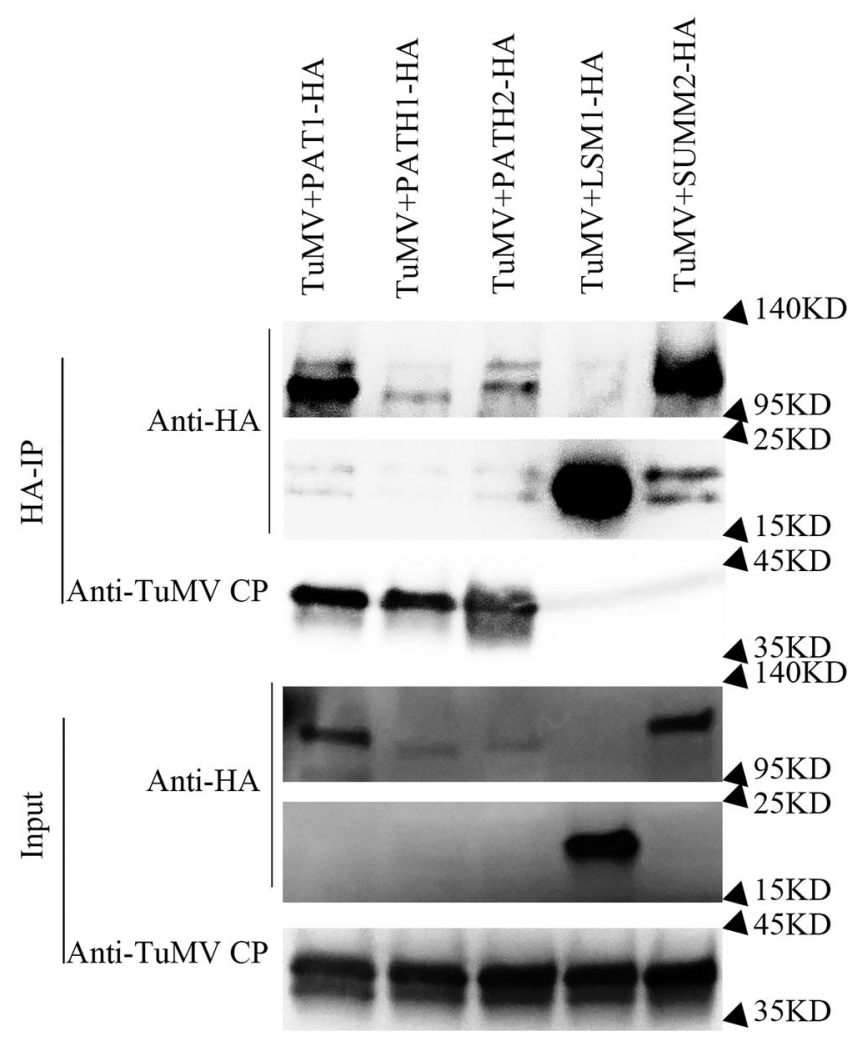

Fig. 4. PATs are in complexes with turnip mosaic virus (TuMV) coat protein (CP). Coimmunoprecipitation between the hemagglutinin (HA)-tagged PAT proteins LSM1, SUMM2, and TuMV CP. Agrobacteria carrying PAT1-HA, PATH1-HA, PATH2-HA, LSM1-HA, and SUMM2-HA were coinfiltrated with the infectious pCB-TuMV-GFP clone in Nicotiana benthamiana and tissue was harvested 3 days postinfiltration. Immunoblots of inputs (lower panels) and HA immunoprecipitates (upper panels) were probed with anti-HA antibodies and anti-TuMV CP antibodies.
PATs in complexes with TuMV CP (Fig. 4), it is likely that TuMV hijacks PAT proteins for processes such as viral assembly.

Although $l s m l a / s m l b$ and pat triple mutants accumulate some TuMV CP compared with eif4e, all three genotypes exhibit resistance to TuMV (Fig. 1). Since PAT1 has been found to interact with eIF4E (Drummond et al. 2011; Wyers et al. 2000), which is targeted by TuMV for viral protein translation, it is possible that PATs and LSM1 function through eIF4E in viral infection. In any event, our results show that LSM1 and the PAT proteins, similarly to $\mathrm{EiF} 4$, are required for TuMV infection.

Given the function of PAT proteins and LSM1 in mRNA decay, the accumulation of target genes due to a nonfunctional mRNA decay machinery in pat triple and $l \mathrm{sm} 1 \mathrm{a} / \mathrm{lsm} 1 \mathrm{~b}$ mutants could affect TuMV infection. However, accumulation of UGT87A2 and ASL9 transcripts upon TuMV infection in Col-0 indicates mRNA decay is compromised by TuMV (Fig. 3). Importantly, viral TuMV RNA is not degraded via the mRNA decay machinery, since the viral RNA degradation rate in pat triple and lsmla/lsmlb mutants was similar to the one observed for Col-0 (Supplementary Fig. S3). This indicates that PAT proteins and LSM1 are recruited for other processes during viral infection. Human PATL1 and LSM1 relocalize from PBs to replication complexes upon $\mathrm{HCV}$ infection and are essential for translation and RNA replication (Scheller et al. 2009). Plant PAT and LSM1 proteins might also play similar roles during potyvirus infection. Nevertheless, only PAT proteins but not LSM1 are detected together with TuMV CP (Fig. 4), this suggests that LSM1 recruitment by TuMV might be indirect and possibly mediated by the PAT proteins.

In summary, we found that the mRNA decapping components LSM1 and PATs are essential for TuMV infection, which is in marked contrast to previously described functions for DCP2 and DHH1. Finally, we show that TuMV hijacks the host decapping machinery by using its $\mathrm{CP}$ to recruit PATs, probably to assist with viral assembly.

Col-0 was used as a control. T-DNA insertion lines At1g79090 (PAT1) pat1 (Salk_040660), At1g12280 (SUMM2) summ2-8 (SAIL_1152A06), double mutants of PAT1 and SUMM2 pat1/ summ2 and of LSM1A and LSM1B lsmla/lsm1b, CRISPR mutants path1/summ2, pat1/path2/summ2, path1/path2/summ2, and pat1/path1/path2/summ2, and the transgenic plants $c$-lsmla (lsmla/lsm1b/LSM1A-GFP) and pat1/Venus-PAT1 have been described (Perea-Resa et al. 2016; Roux et al. 2015; Zhang et al. 2012; Zuo et al. 2019). The T-DNA insertion line for At3g61240 (DHH1) dhhl (Salk_016921) is from Nottingham Arabidopsis Stock Centre (U.K.), and primers for genotyping are listed in Supplementary Table S1. Plants were grown in $9 \times 9$-cm pots at $21^{\circ} \mathrm{C}$ with $8 \mathrm{~h}$ of light and $16 \mathrm{~h}$ of dark.

The infectious pCB-TuMV-GFP clone in Agrobacteria tumefaciens used in this study has been described (Garcia-Ruiz et al. 2010). Viral particles purified from TuMV-GFP (Garcia-Ruiz et al. 2010) infected Nicotiana benthamiana plants were mechanically rubbed on the first true leaves of 3-week-old Arabidopsis plants using a sponge and spatula with Carborundum. Infected plants were identified by visual inspection of local leaf GFP signal, typical disease symptoms, and by quantification of TuMV $\mathrm{CP}$ from new emerging leaves with anti-CP antibodies.

Viral RNA transcription was blocked by cordycepin treatment as previously described (Xu and Chua 2009). The 14-dpi TuMVinfected plants were incubated in liquid phosphate buffered saline (PBS) for $2 \mathrm{~h}$, then, the same amount of PBS or PBS + 1 $\mathrm{mM}$ cordycepin (Sigma-Aldrich) were added and incubated for $1 \mathrm{hr}$ or $3 \mathrm{hr}$ before collected for RNA extraction.

A. tumefaciens strains carrying HA-tagged PAT proteins, LSM1 and SUMM2 fusions, and pCB-TuMV-GFP were grown in yeast-extract peptone medium supplemented with appropriate antibiotics overnight. Cells were centrifuged and were resuspended in 
buffer (10 mM MgCl $2,10 \mathrm{mM}$ MES-K (pH 5.6), and $100 \mu \mathrm{M}$ acetosyringone) to an optical density at $600 \mathrm{~nm}=0.8$. A. tumefaciens strains carrying pCB-TuMV-GFP and PAT1-HA, PATH1-HA, and PATH2-HA were mixed 1:1 and were infiltrated into 3week-old $N$. benthamiana leaves. Leaf samples for protein extraction and immunoprecipitation were collected 3 days postinfiltration. Tissues for extraction were ground in liquid nitrogen and immunoprecipitation buffer $(50 \mathrm{mM}$ Tris- $\mathrm{HCl}, \mathrm{pH} 7.5,150$ $\mathrm{mM} \mathrm{NaCl}, 0.1 \%$ [vol/vol] NP40, $10 \mathrm{mM}$ dithiothreitol, 5\% [vol/vol] glycerol, $1 \mathrm{mM}$ EDTA, protease inhibitor cocktail [Roche], Phosstop [Roche]) was added at $2 \mathrm{ml}$ per gram of tissue powder. Following a 20 -min centrifugation at $4{ }^{\circ} \mathrm{C}$ and 13,000 rpm using an Eppendorf Centrifuge 5417R, sample supernatants were adjusted to $2 \mathrm{mg}$ of protein per milliliter and were incubated $4 \mathrm{~h}$ at $4{ }^{\circ} \mathrm{C}$ with monoclonal anti-HA agarose antibody (Sigma). Beads were washed four times with wash buffer $(20 \mathrm{mM}$ Tris, $\mathrm{pH} 7.5,150 \mathrm{M} \mathrm{NaCl}, 0.1 \%$ [vol/vol] NP40 before adding 4x sodium dodecyl sulfate [SDS] buffer [Novex]) and were denatured by heating at $95^{\circ} \mathrm{C}$ for $5 \mathrm{~min}$.

Tissue samples were ground in liquid nitrogen and $4 \times$ SDS buffer was then added and samples were heated at $95^{\circ} \mathrm{C}$ for $5 \mathrm{~min}$, were cooled to room temperature for $10 \mathrm{~min}$, were centrifuged $5 \mathrm{~min}$ at 13,000 rpm using the Eppendorf Centrifuge 5417R, and supernatants were separated on $10 \%$ SDS-polyacrylamide gel electrophoresis gels, were electroblotted onto polyvinylidene diflouride membrane (GE Healthcare), were blocked in 5\% (wt/ vol) bovine serum ablumin in TBS-Tween 20 (TBS-T; $0.1 \%$, $\mathrm{vol} / \mathrm{vol}$ ), and were incubated for $1 \mathrm{~h}$ to overnight with primary antibody (anti-TuMV CP 1:1.000, anti-HA 1:1,000). Membranes were washed $3 \times$ for $10 \mathrm{~min}$ in TBS-T $(0.1 \%)$ before $1 \mathrm{~h}$ of incubation in secondary antibodies (anti-rabbit or -mouse horseradish peroxidase or ammonium phosphate [AP] conjugate [Promega], 1:5,000]). Chemiluminescent substrate (ECL Plus [Pierce]) was applied before camera detection. For AP-conjugated primary antibodies, membranes were incubated in NBT/BCIP (Roche) until bands were visible.

Total RNA was extracted with TRIzol Reagent (Thermo Scientific), $1 \mu \mathrm{g}$ of total RNA were treated with DNAse I (Thermo Scientific) and were reverse-transcribed into cDNA using a RevertAid first strand cDNA synthesis kit (Thermo Scientific). The ACT2 gene was used as an internal control. Quantitative PCR assays with SYBR Green master mix were performed on a Bio-RAD CFX96 system (Thermo Scientific). Primers are listed in Supplementary Table S1. All experiments were performed at least three times each in technical triplicates.

Statistical details of experiments are reported in the figures and captions. Statistical significance between groups was determined by one-way analysis of variance followed by Duncan's new multiple range test and Tukey's honest significance test with Systat software.

\section{ACKNOWLEDGMENTS}

We thank J. Salinas for $l s m 1 a / l s m 1 b$ and $c$-lsmla seeds, J. Carrington for providing the TuMV-GFP infectious clone, and F. Rabenstein for the TuMV antiserum. A special thanks to J. Mundy for advice throughout the project and for critically reading the manuscript.

\section{LITERATURE CITED}

Charenton, C., Gaudon-Plesse, C., Fourati, Z., Taverniti, V., Back, R., Kolesnikova, O., Séraphin, B., and Graille, M. 2017. A unique surface on Pat1 C-terminal domain directly interacts with Dcp2 decapping enzyme and Xrn1 5'-3' mRNA exonuclease in yeast. Proc. Natl. Acad. Sci. U.S.A. 114:E9493-E9501.

Checkley, M. A., Nagashima, K., Lockett, S. J., Nyswaner, K. M., and Garfinkel, D. J. 2010. P-body components are required for Ty1 retrotransposition during assembly of retrotransposition-competent viruslike particles. Mol. Cell. Biol. 30:382-398.
Cheng, X., and Wang, A. 2017. The potyvirus silencing suppressor protein VPg mediates degradation of SGS3 via ubiquitination and autophagy pathways. J. Virol. 91:01478-16.

Chowdhury, A., Kalurupalle, S., and Tharun, S. 2014. Pat1 contributes to the RNA binding activity of the Lsm1-7-Pat1 complex. RNA 20:1465-1475.

Ding, S. W., and Voinnet, O. 2007. Antiviral immunity directed by small RNAs. Cell 130:413-426.

Drummond, S. P., Hildyard, J., Firczuk, H., Reamtong, O., Li, N., Kannambath, S., Claydon, A. J., Beynon, R. J., Eyers, C. E., and McCarthy, J. E. G. 2011. Diauxic shift-dependent relocalization of decapping activators Dhh1 and Pat1 to polysomal complexes. Nucleic Acids Res. 39:7764-7774.

Duprat, A., Caranta, C., Revers, F., Menand, B., Browning, K. S., and Robaglia, C. 2002. The Arabidopsis eukaryotic initiation factor (iso)4E is dispensable for plant growth but required for susceptibility to potyviruses. Plant J. 32:927-934.

Fauquet, C. M., and Fargette, D. 2005. International Committee on Taxonomy of Viruses and the 3,142 unassigned species. Virol. J. 2:64.

Garcia-Ruiz, H., Carbonell, A., Hoyer, J. S., Fahlgren, N., Gilbert, K. B., Takeda, A., Giampetruzzi, A., Garcia Ruiz, M. T., McGinn, M. G., Lowery, N., Martinez Baladejo, M. T., and Carrington, J. C. 2015. Roles and programming of Arabidopsis ARGONAUTE proteins during Turnip mosaic virus infection. PLoS Pathog. 11:e1004755.

Garcia-Ruiz, H., Takeda, A., Chapman, E. J., Sullivan, C. M., Fahlgren, N., Brempelis, K. J., and Carrington, J. C. 2010. Arabidopsis RNAdependent RNA polymerases and dicer-like proteins in antiviral defense and small interfering RNA biogenesis during Turnip mosaic virus infection. Plant Cell 22:481-496.

Garneau, N. L., Wilusz, J., and Wilusz, C. J. 2007. The highways and byways of mRNA decay. Nat. Rev. Mol. Cell Biol. 8:113-126.

Hafrén, A., Lõhmus, A., and Mäkinen, K. 2015. Formation of Potato virus $A$-induced RNA granules and viral translation are interrelated processes required for optimal virus accumulation. PLoS Pathog. 11: e1005314.

Jungfleisch, J., Chowdhury, A., Alves-Rodrigues, I., Tharun, S., and Díez, J. 2015. The Lsm1-7-Pat1 complex promotes viral RNA translation and replication by differential mechanisms. RNA 21:1469-1479.

Kung, Y. J., Lin, P. C., Yeh, S. D., Hong, S. F., Chua, N. H., Liu, L. Y., Lin, C. P., Huang, Y. H., Wu, H. W., Chen, C. C., and Lin, S. S. 2014. Genetic analyses of the FRNK motif function of Turnip mosaic virus uncover multiple and potentially interactive pathways of crossprotection. Mol. Plant-Microbe Interact. 27:944-955.

Li, F., and Wang, A. 2018. RNA decay is an antiviral defense in plants that is counteracted by viral RNA silencing suppressors. PLoS Pathog. 14:e1007228.

Lin, S. S., Wu, H. W., Jan, F. J., Hou, R. F., and Yeh, S. D. 2007. Modifications of the helper component-protease of Zucchini yellow mosaic virus for generation of attenuated mutants for cross protection against severe infection. Phytopathology 97:287-296.

Olspert, A., Chung, B. Y. W., Atkins, J. F., Carr, J. P., and Firth, A. E. 2015. Transcriptional slippage in the positive-sense RNA virus family Potyviridae. EMBO Rep. 16:995-1004.

Ozgur, S., Chekulaeva, M., and Stoecklin, G. 2010. Human Pat1b connects deadenylation with mRNA decapping and controls the assembly of processing bodies. Mol. Cell. Biol. 30:4308-4323.

Perea-Resa, C., Carrasco-López, C., Catalá, R., Turečková, V., Novak, O., Zhang, W., Sieburth, L., Jiménez-Gómez, J. M., and Salinas, J. 2016. The LSM1-7 complex differentially regulates Arabidopsis tolerance to abiotic stress conditions by promoting selective mRNA decapping. Plant Cell 28:505-520.

Pilkington, G. R., and Parker, R. 2008. Pat1 contains distinct functional domains that promote P-body assembly and activation of decapping. Mol. Cell. Biol. 28:1298-1312.

Poque, S., Wu, H. W., Huang, C. H., Cheng, H. W., Hu, W. C., Yang, J. Y., Wang, D., and Yeh, S. D. 2018. Potyviral gene-silencing suppressor HCPro interacts with salicylic acid (SA)-binding protein 3 to weaken SA-mediated defense responses. Mol. Plant-Microbe Interact. 31:86-100.

Pumplin, N., and Voinnet, O. 2013. RNA silencing suppression by plant pathogens: Defence, counter-defence and counter-counter-defence. Nat. Rev. Microbiol. 11:745-760.

Qu, F., Ye, X., and Morris, T. J. 2008. Arabidopsis DRB4, AGO1, AGO7, and RDR6 participate in a DCL4-initiated antiviral RNA silencing pathway negatively regulated by DCL1. Proc. Natl. Acad. Sci. U.S.A. 105: 14732-14737.

Rodamilans, B., Valli, A., Mingot, A., San León, D., Baulcombe, D., López-Moya, J. J., and García, J. A. 2015. RNA polymerase slippage as a mechanism for the production of frameshift gene products in plant viruses of the potyviridae family. J. Virol. 89:6965-6967. 
Roux, M. E., Rasmussen, M. W., Palma, K., Lolle, S., Regué, A. M. Bethke, G., Glazebrook, J., Zhang, W., Sieburth, L., Larsen, M. R., Mundy, J., and Petersen, M. 2015. The mRNA decay factor PAT1 functions in a pathway including MAP kinase 4 and immune receptor SUMM2. EMBO J. 34:593-608.

Scheller, N., Mina, L. B., Galão, R. P., Chari, A., Giménez-Barcons, M., Noueiry, A., Fischer, U., Meyerhans, A., and Díez, J. 2009. Translation and replication of hepatitis $\mathrm{C}$ virus genomic RNA depends on ancient cellular proteins that control mRNA fates. Proc. Natl. Acad. Sci. U.S.A. 106:13517-13522.

Tharun, S., He, W., Mayes, A. E., Lennertz, P., Beggs, J. D., and Parker, R. 2000. Yeast Sm-like proteins function in mRNA decapping and decay. Nature 404:515-518.

Tharun, S., and Parker, R. 2001. Targeting an mRNA for decapping: Displacement of translation factors and association of the Lsm1p-7p complex on deadenylated yeast mRNAs. Mol. Cell 8:1075-1083.

Thran, M., Link, K., and Sonnewald, U. 2012. The Arabidopsis DCP2 gene is required for proper mRNA turnover and prevents transgene silencing in Arabidopsis. Plant J. 72:368-377.

Urcuqui-Inchima, S., Haenni, A. L., and Bernardi, F. 2001. Potyvirus proteins: A wealth of functions. Virus Res. 74:157-175.

Vogel, F., Hofius, D., Paulus, K. E., Jungkunz, I., and Sonnewald, U. 2011. The second face of a known player: Arabidopsis silencing suppressor AtXRN4 acts organ-specifically. New Phytol. 189:484-493.

White, K. A. 2015. The polymerase slips and PIPO exists. EMBO Rep. 16:885-886.
Wyers, F., Minet, M., Dufour, M. E., Vo, L. T. A., and Lacroute, F. 2000. Deletion of the PAT1 gene affects translation initiation and suppresses a PAB1 gene deletion in yeast. Mol. Cell. Biol. 20: 3538-3549.

Wylie, S. J., Adams, M., Chalam, C., Kreuze, J., López-Moya, J. J., Ohshima, K., Praveen, S., Rabenstein, F., Stenger, D., Wang, A., Zerbini, F. M., and Ictv Report Consortium. 2017. ICTV Virus Taxonomy Profile: Potyviridae. J. Gen. Virol. 98:352-354.

$\mathrm{Xu}$, J., and Chua, N. H. 2009. Arabidopsis decapping 5 is required for mRNA decapping, P-body formation, and translational repression during postembryonic development. Plant Cell 21:3270-3279.

Xu, J., and Chua, N. H. 2011. Processing bodies and plant development. Curr. Opin. Plant Biol. 14:88-93.

Zhang, Z., Wu, Y., Gao, M., Zhang, J., Kong, Q., Liu, Y., Ba, H., Zhou, J., and Zhang, Y. 2012. Disruption of PAMP-induced MAP kinase cascade by a Pseudomonas syringae effector activates plant immunity mediated by the NB-LRR protein SUMM2. Cell Host Microbe 11: 253-263.

Zuo, Z., Roux, M. E., Rodriguez, E., Chevalier, J. R., Dagdas, Y., Yamashino, T., and Petersen, M. 2019. mRNA decapping machinery targets transcripts of the LBD3/ASL9 transcription factor to authorize formation of apical hook and lateral roots in Arabidopsis. bioRxiv:834465.

Zuo, Z., Roux, M. E., Saemundsson, H. P., Müller, M., Munne Bosch, S., and Petersen, M. 2021. The Arabidopsis thaliana mRNA decay factor PAT1 functions in osmotic stress responses and decaps ABA-responsive genes. FEBS Lett. 595:253-263. 